\title{
Earthquake source model using strong motion displacement as response of finite elastic media
}

\author{
R N IYENGAR* and Shailesh KR AgRAWAL** \\ *Department of Civil Engineering, Indian Institute of Science, Bangalore 560 012, India. \\ e-mail:rni@civil.iisc.ernet.in \\ **Central Building Research Institute, Roorkee, India.
}

\begin{abstract}
The strong motion displacement records available during an earthquake can be treated as the response of the earth as the a structural system to unknown forces acting at unknown locations. Thus, if the part of the earth participating in ground motion is modelled as a known finite elastic medium, one can attempt to model the source location and forces generated during an earthquake as an inverse problem in structural dynamics. Based on this analogy, a simple model for the basic earthquake source is proposed. The unknown source is assumed to be a sequence of impulses acting at locations yet to be found. These unknown impulses and their locations are found using the normal mode expansion along with a minimization of mean square error. The medium is assumed to be finite, elastic, homogeneous, layered and horizontal with a specific set of boundary conditions. Detailed results are obtained for Uttarkashi earthquake. The impulse locations exhibit a linear structure closely associated with the causative fault. The results obtained are shown to be in good agreement with reported values. The proposed engineering model is then used to simulate the acceleration time histories at a few recording stations. The earthquake source in terms of a sequence of impulses acting at different locations is applied on a 2D finite elastic medium and acceleration time histories are found using finite element methods. The synthetic accelerations obtained are in close match with the recorded accelerations.
\end{abstract}

\section{Introduction}

Simulation of ground motion to possible earthquakes is essential in the aseismic design of man made structures. In literature, three broad approaches are available for this purpose. The first is the purely empirical method of modelling ground motions as a stochastic process and to describe the ground motion in terms of various statistical parameters and frequency spectra (Shinozuka and Sato 1967; Iyengar and Iyengar 1969). The second approach is based on acceptable source mechanism and wave propagation in horizontally layered elastic media (Khattri et al 1994; Yu et al 1995). There are also hybrid methods, called empirical Green's function approach, wherein observed acceler- ograms from small earthquakes are judiciously combined to simulate surface motion from larger events (Kumar et al 1999). In the present paper, a method is proposed for representing recorded strong motion displacement time histories as response of the near source region modelled as a finite two dimensional elastic medium. For the source, a simple external force mechanism is postulated. The magnitude and location of this source is found by normal mode analysis of the near source region coupled with an error minimization procedure. The source thus determined is demonstrated to be capable of simulating recorded ground acceleration time histories. Preliminary results are presented for the case of data recorded during the Uttarkashi earthquake of 20th October 1991.

Keywords. Strong motion; array data; normal mode; elastic medium; earthquake source; mean square error. 


\section{Strong motion displacement}

The ground motion at a station during an earthquake is a function of the details of the source, the intervening medium and distance from the source. From a basic point of view, the whole earth would participate in the event. However, strong motion is localized and it is the near source region which primarily takes part in this motion. Seen from this perspective, it is natural to model strong motion array data as the surface response of the near source region to unknown disturbances occurring within the medium at unknown locations. The features of strong motion acceleration, velocity and displacement are well known. In figure 1, a few near field displacement records of the Uttarkashi event are presented. These are low frequency transient signals with a predominant frequency of about $0.07 \mathrm{~Hz}$ as seen from the Fourier spectra shown in figure 2 . The spectra indicate that for modelling purposes, the highest frequency can be taken to be about $0.5 \mathrm{~Hz}$. It is to be noted here that these displacement records are obtained by double integration of recorded accelerograms and also their absolute starting time are not known. Thus, what can be inferred from these response time histories are the gross features of the source.

\section{Limited area regional model}

There are several models available in the literature for representing the intervening path between the source and the site. The simplest of the models use horizontally layered media with different densities, wave velocities and damping factors. For the Uttarkashi region of the Himalayas, previously, Khattri (1993), Khattri et al (1994) have proposed such layered media models. The layout of the strong motion array, which, recorded the Uttarkashi earthquake of 1991 (Chandrasekaran and Das 1995) is shown in figure 3 . The fault dimensions for a 6.5 magnitude earthquake are expected to be of the order of $20 \times 20 \mathrm{~km}$. The depth of the focus for the event is reported to be of the order of $10 \mathrm{~km}$. Thus for constraining the source, the near source region has to have dimensions of the order of $100 \times 100 \times 50 \mathrm{~km}$. The strong motion records are essentially the surface displacements of such an elastic body for the forces arising at the fault plane. If the properties of this medium are known, source determination reduces to finding a system of consistent forces along with their locations for a given set of the measured surface displacements.

In the present exploratory work, the near source region is further taken to be two-dimensional, such that a few of the recording stations lie on the top edge of the medium. The excitation source is represented by a sequence of impulses in the two orthogonal directions, the magnitude and location of which are yet to be determined. The equations of motion of the medium, for layer $n(n=1,2, \ldots N)$ subjected to a force $\left[f_{x}(t), f_{z}(t)\right]$ at location $\left(c_{s x}, c_{s z}\right)$ applied at instant $t_{s}(s=0,1,2 \ldots m)$ are (Ewing et al 1957)

$$
\begin{aligned}
& \rho_{n} \ddot{u}_{n}+c_{n} \dot{u}_{n}-f_{x}(t) \delta\left(x-c_{\ell x}\right) \delta\left(z-c_{\ell z}\right) \\
& =\left(\lambda_{n}+\mu_{n}\right) \frac{\partial}{\partial x}\left[\frac{\partial u_{n}}{\partial x}+\frac{\partial w_{n}}{\partial z}\right]+\mu_{n} \nabla^{2} u_{n}, \\
& \rho_{n} \ddot{w}_{n}+c_{n} \dot{w}_{n}-f_{z}(t) \delta\left(x-c_{\ell x}\right) \delta\left(z-c_{\ell z}\right) \\
& =\left(\lambda_{n}+\mu_{n}\right) \frac{\partial}{\partial y}\left[\frac{\partial w_{n}}{\partial x}+\frac{\partial u_{n}}{\partial z}\right]+\mu_{n} \nabla^{2} w_{n} .
\end{aligned}
$$

The boundary conditions are: the bottom edge $z=H$ is fixed and the top edge is stress free. At the interfaces, the displacements and the stresses are continuous. The lateral boundary conditions at $x=0$ and $x=L$ are taken as $u=\frac{\partial w}{\partial x}=0$ for all the layers. It is expected that if the lateral boundaries are far away from the epicentre, the boundary conditions there may not affect the final results. The normal modes (eigen functions) of the medium $U_{j k}(x, z) ; W_{j k}(x, z)$ along with natural frequencies $\omega_{j k}$ in the mode $(j=1,2 \ldots ; k=1,2 \ldots)$ can be found out by well known methods. Such analysis has been previously presented by Iyengar and Rao (1975) for a single layer. This solution can be easily extended to the present case also and hence the details are not given here. The eigen functions satisfy the orthogonality condition

$$
\begin{aligned}
\int_{0}^{H} \int_{0}^{L} \rho\left[U_{j k} U_{l m}+W_{j k} W_{l m}\right] \mathrm{d} x \mathrm{~d} z & =0 ; \quad j \neq l ; k \neq m \\
& =M_{j k} ; \quad \text { otherwise }
\end{aligned}
$$

Apart from the eigen functions and eigen values, one needs to know the dissipation mechanism in the medium. Reliable $Q$-factors for the Himalayan region are not available. With this in view, here the simplest modal damping model is used such that $c_{n} / \rho_{n}$ $=2 \eta_{j k} \omega_{j k}$ and $\eta_{j k}$ is taken as 0.1 for the medium in all the modes. This is not unreasonable, since in engineering literature, the soil and rock damping coefficient is generally taken to be 5 to $15 \%$ of the critical value.

\section{Source location}

Let the medium be subjected to a sequence of impulses $\left(I_{\ell x}, I_{\ell z}\right)$ applied at known time instants $t=t_{l}$ at unknown locations $\left(c_{\ell x}, c_{\ell z}\right)(l=0,1,2 \ldots m)$. The responses $u(x, 0 ; t)$ and $w(x, 0 ; t)$ are known at a few points $\left(x=x_{i}, z=0\right)(i=1,2 \ldots p)$ on the surface in the time interval $(0, T)$. The source location problem is to estimate the points $\left(c_{l x}, c_{l z}\right)$ and the corresponding impulses $\left(I_{\ell x}, I_{\ell z}\right)$. For a given impulse, the equations of motion given by equations (1) and (2) 

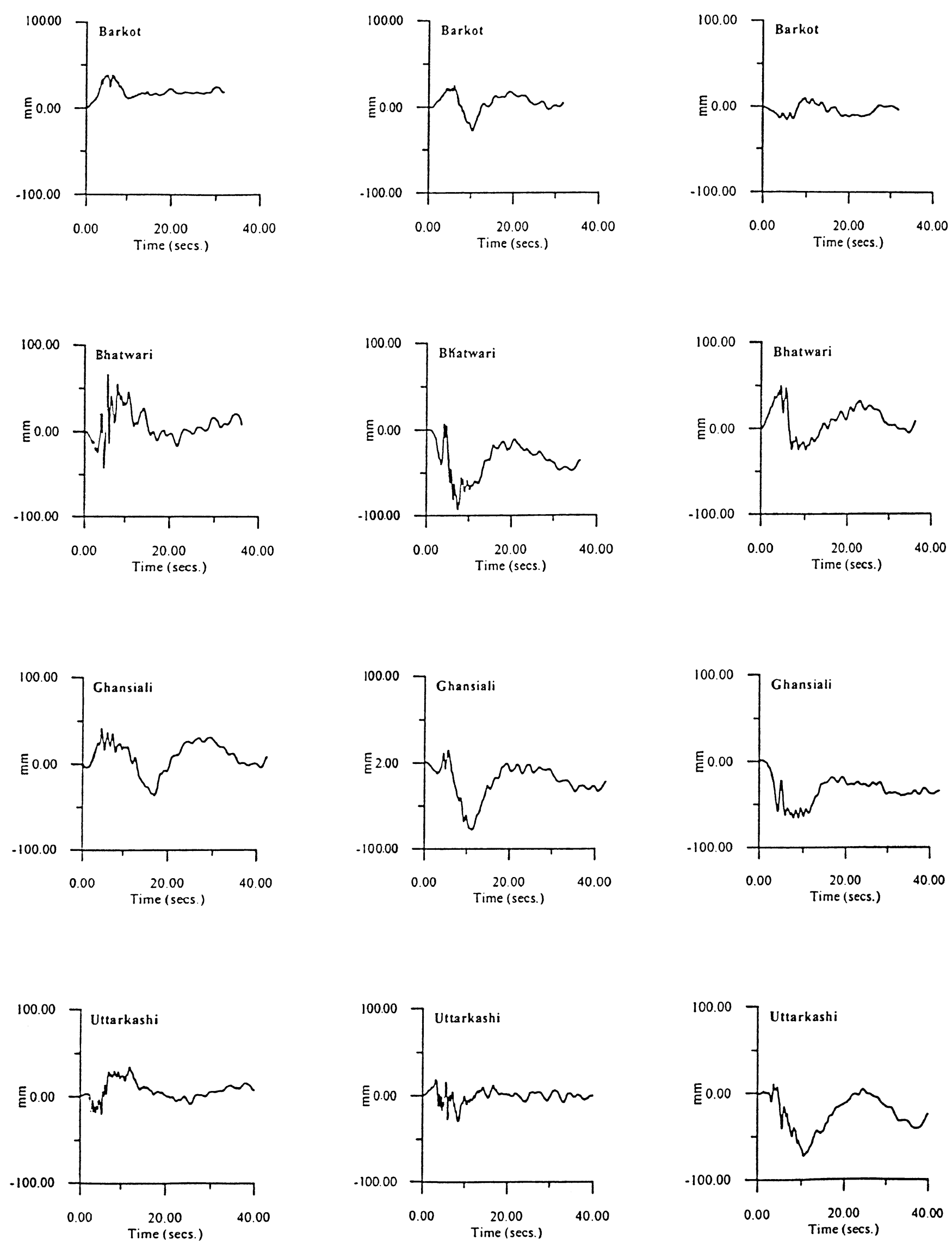

Figure 1. Displacement time histories for Uttarkashi earthquake (NS, EW and vertical components). 


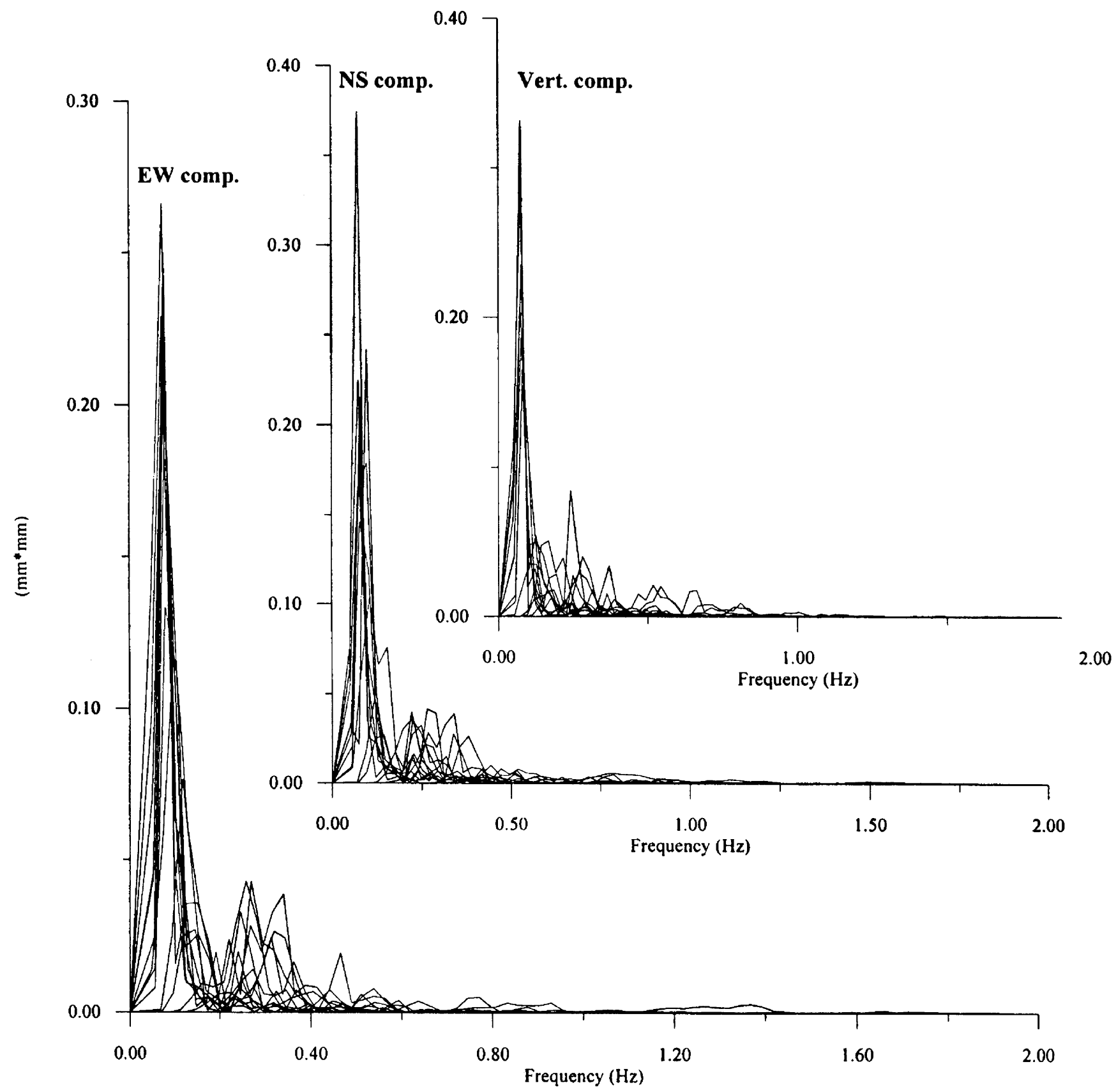

Figure 2. Fourier spectra of near source displacement (Uttarkashi earthquake).

can be solved by the normal mode method in terms of the eigen functions as

$$
\begin{aligned}
u(x, z ; t) & =\sum_{j} \sum_{k} a_{j k}(t) U_{j k}(x, z), \\
w(x, z ; t) & =\sum_{j} \sum_{k} a_{j k}(t) W_{j k}(x, z) .
\end{aligned}
$$

With the help of the orthogonality condition of equation (3), the equation for $a_{j k}(t)$ will be

$$
\ddot{a}_{j k}+2 \eta \omega_{j k} \dot{a}_{j k}+\omega_{j k}^{2} a_{j k}=F_{j k} / M_{j k}
$$

where,

$$
M_{j k}=\int_{0}^{H} \int_{0}^{L} \rho\left[U_{j k}^{2}+W_{j k}^{2}\right] \mathrm{d} x \mathrm{~d} z
$$

$$
F_{j k}(t)=f_{x}(t) U_{j k}\left(c_{\ell x}, c_{\ell z}\right)+f_{z}(t) W_{j k}\left(c_{\ell x}, c_{\ell x}\right) .
$$

If the force $\left(f_{x}, f_{z}\right)$ is taken as a sequence of impulses $\left(I_{\ell x}, I_{\ell z}\right)$ applied at time $t=t_{\ell}$, it easily follows that in the time interval $\left(t_{s} \leq t<t_{s+1}\right)$

$$
\begin{aligned}
a_{j k}(t)= & \left(1 / M_{j k}\right) \sum_{l=0}^{s}\left[I_{\ell x} U_{j k}\left(c_{\ell x}, c_{\ell z}\right)\right. \\
& \left.+I_{\ell z} W_{j k}\left(c_{\ell x}, c_{\ell z}\right)\right] h_{j k}\left(t-t_{\ell}\right), \\
h_{j k}(t) & =\left(1 / \omega_{j k}\right) e^{-\eta_{j k} \omega_{j k} t} \sin \left(\varpi_{j k} t\right),
\end{aligned}
$$




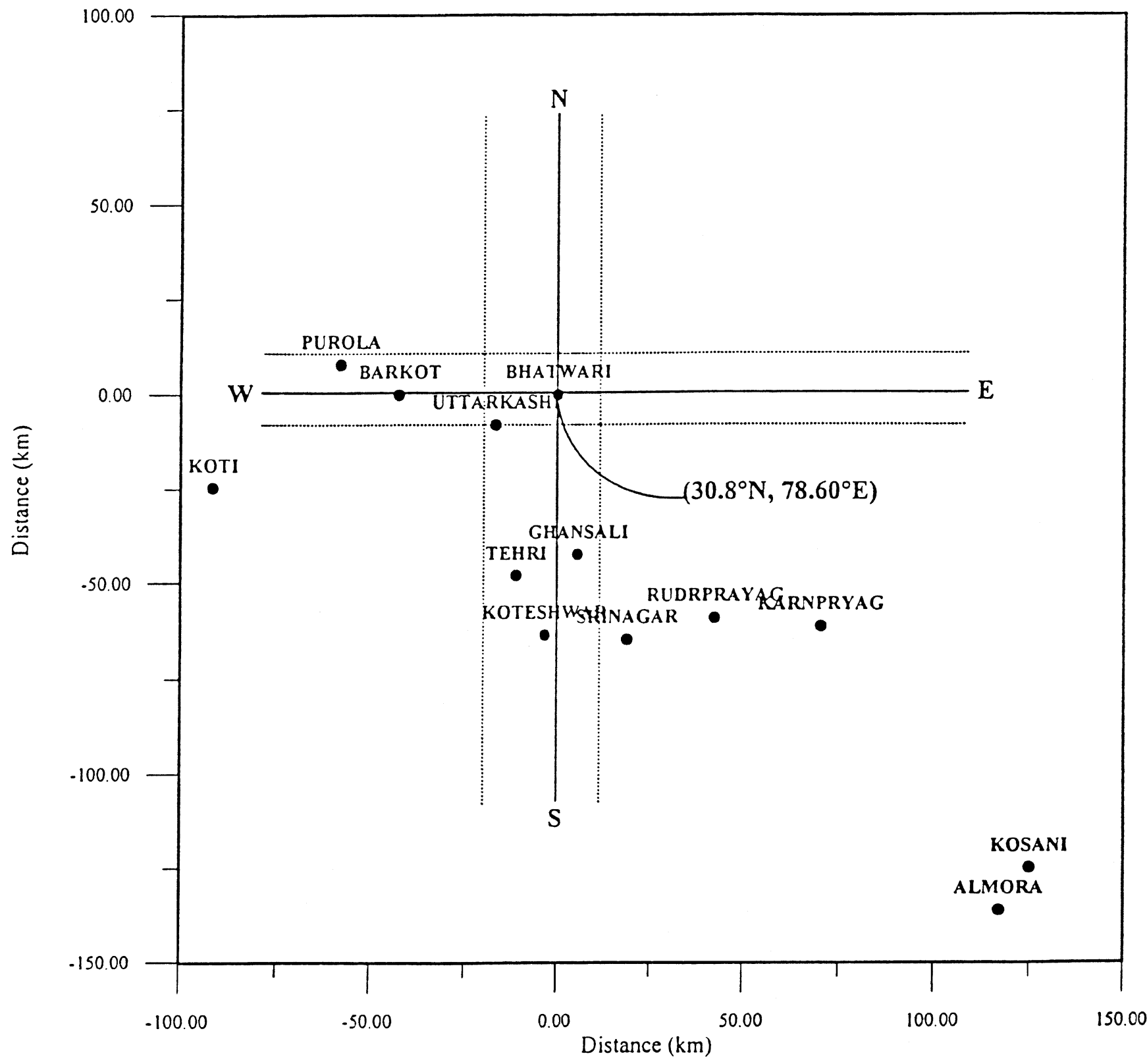

Figure 3. Uttarkashi SM array layout.

Now, the surface displacement at station $i(i=1,2 \ldots p)$ can be expressed after some algebra as

$$
\begin{gathered}
u_{i}(t)=\sum_{l=0}^{s} I_{\ell x} P_{u, i l}(t)+I_{\ell z} Q_{u, i l}(t), \\
P_{u, i l}=\sum_{j} \sum_{k} M_{j k}^{-1} U_{j k}\left(x_{i}, 0\right) U_{j k}\left(c_{\ell x}, c_{\ell z}\right) h_{j k}\left(t-t_{\ell}\right), \\
Q_{u, i l}=\sum_{j} \sum_{k} M_{j k}^{-1} U_{j k}\left(x_{i}, 0\right) W_{j k}\left(c_{\ell x}, c_{\ell z}\right) h_{j k}\left(t-t_{\ell}\right) .
\end{gathered}
$$

Similarly,

$$
w_{i}(t)=\sum_{l=0}^{s} I_{\ell x} P_{w, i l}(t)+I_{\ell z} Q_{w, i l}(t),
$$

$$
\begin{gathered}
P_{w, i l}=\sum_{j} \sum_{k} M_{j k}^{-1} W_{j k}\left(x_{i}, 0\right) U_{j k}\left(c_{\ell x}, c_{\ell z}\right) h_{j k}\left(t-t_{\ell}\right) \\
Q_{w, i l}= \\
\quad \sum_{j} \sum_{k} M_{j k}^{-1} W_{j k}\left(x_{i}, 0\right) \\
\\
\times W_{j k}\left(c_{\ell x}, c_{\ell z}\right) h_{j k}\left(t-t_{\ell}\right) .
\end{gathered}
$$

The number of terms in the summation over $l$ in equations (12) and (15) depends on the time interval under consideration. For example in $\left(0 \leq t<t_{1}\right)$, only one term with $\left(I_{o x}, I_{o z}\right)$ will be present.

In any time interval $\left(t_{s} \leq t<t_{s+1}\right)$, there are four unknowns namely $\left(I_{s x}, I_{s z}\right)$ and $\left(c_{s x}, c_{s z}\right)$. Since $u_{i}(t)$ and $w_{i}(t)$ in equations (12) and (15) are measured and known for all time $t$, the unknowns on the right hand side of these equations can be found by minimizing the 
14

$R N$ Iyengar and $S K r$ Agrawal
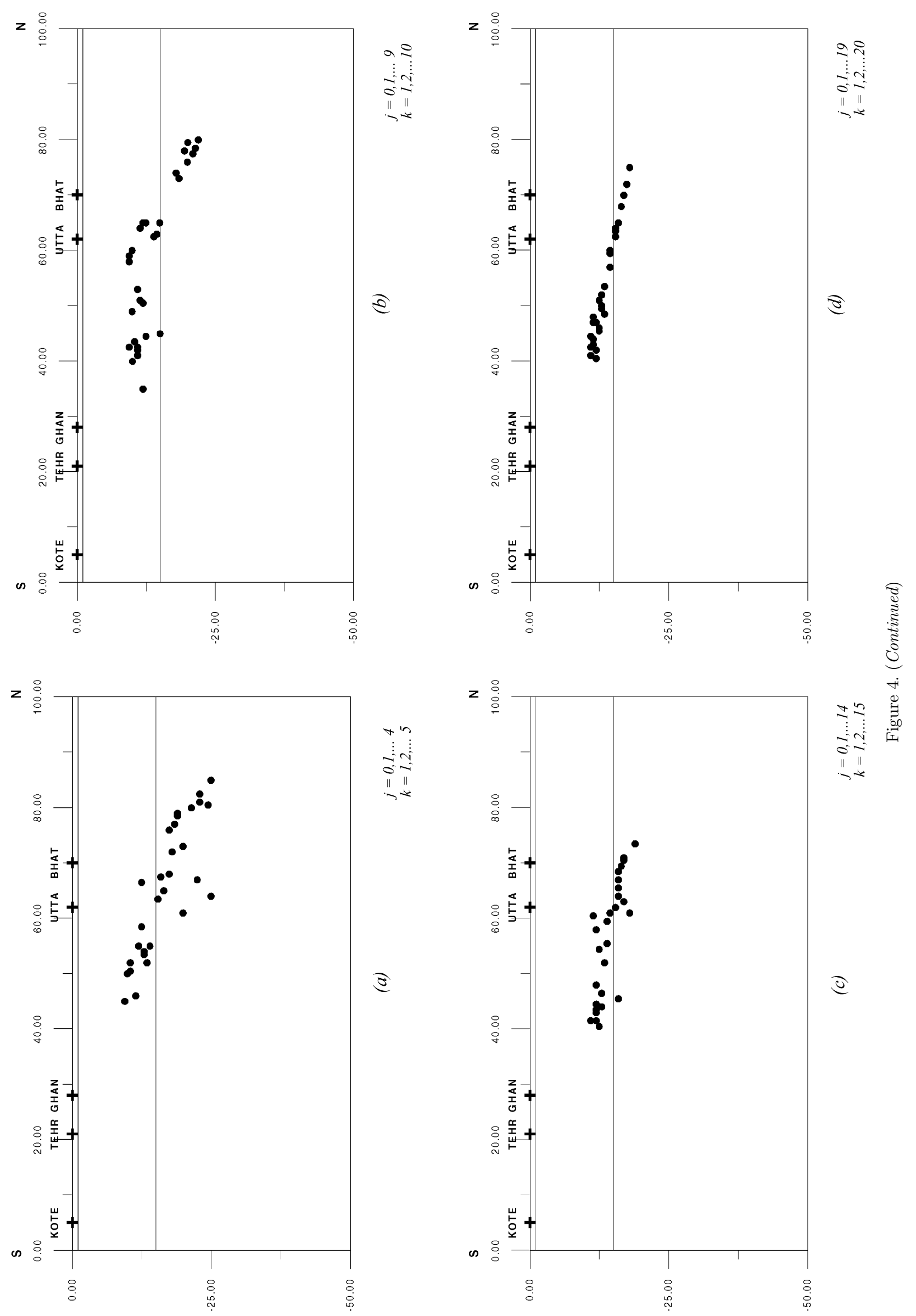


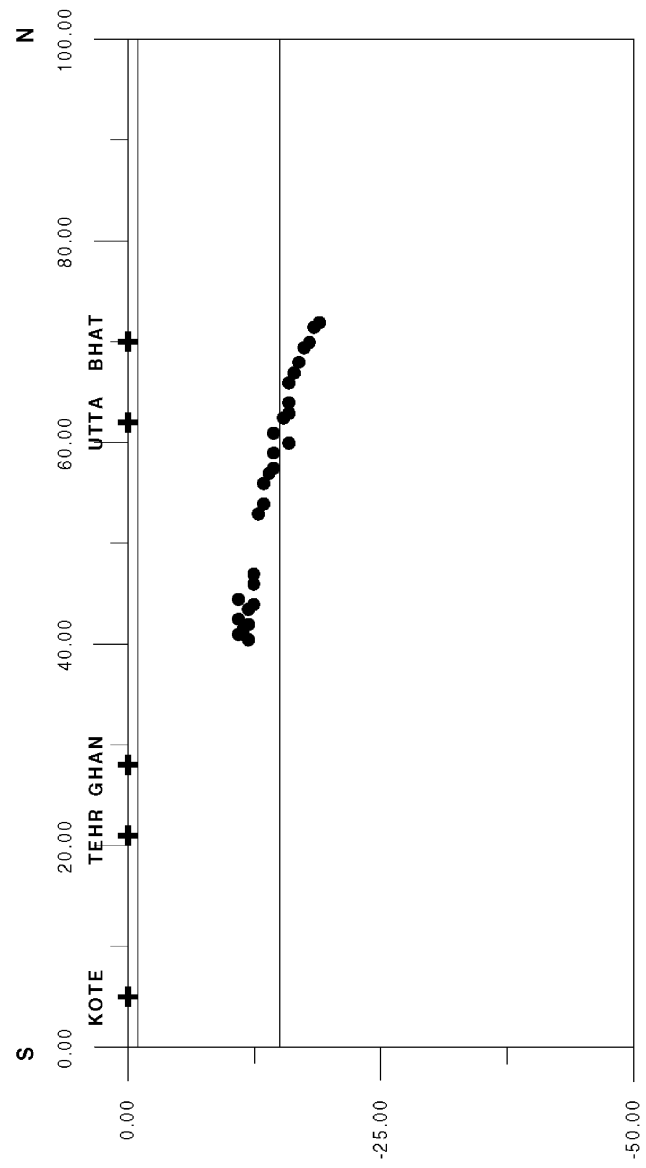

רิ?

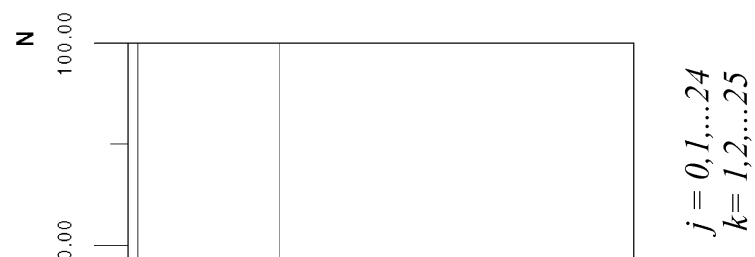

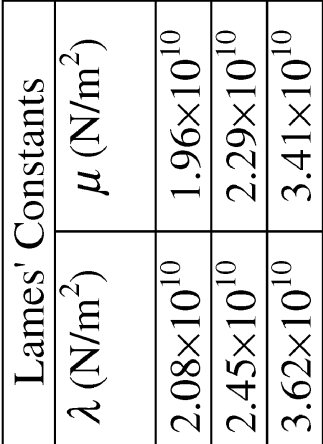

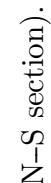

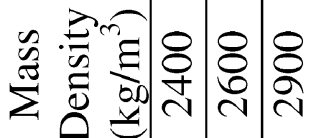

更

8

خิ ฺ

- i

$\|$

(2)

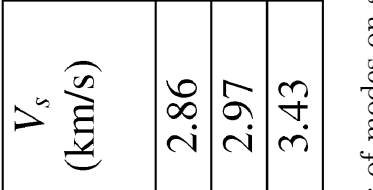
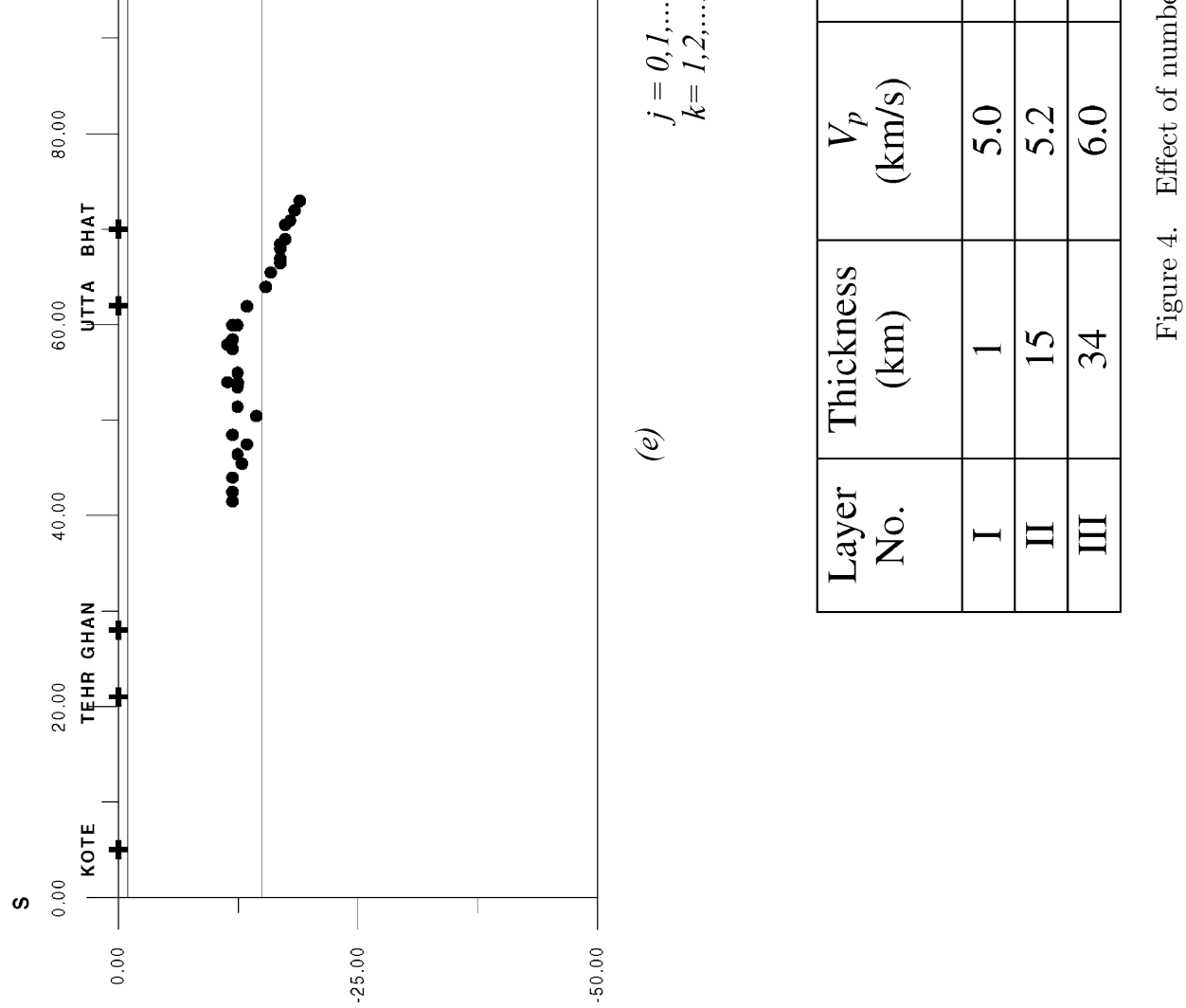


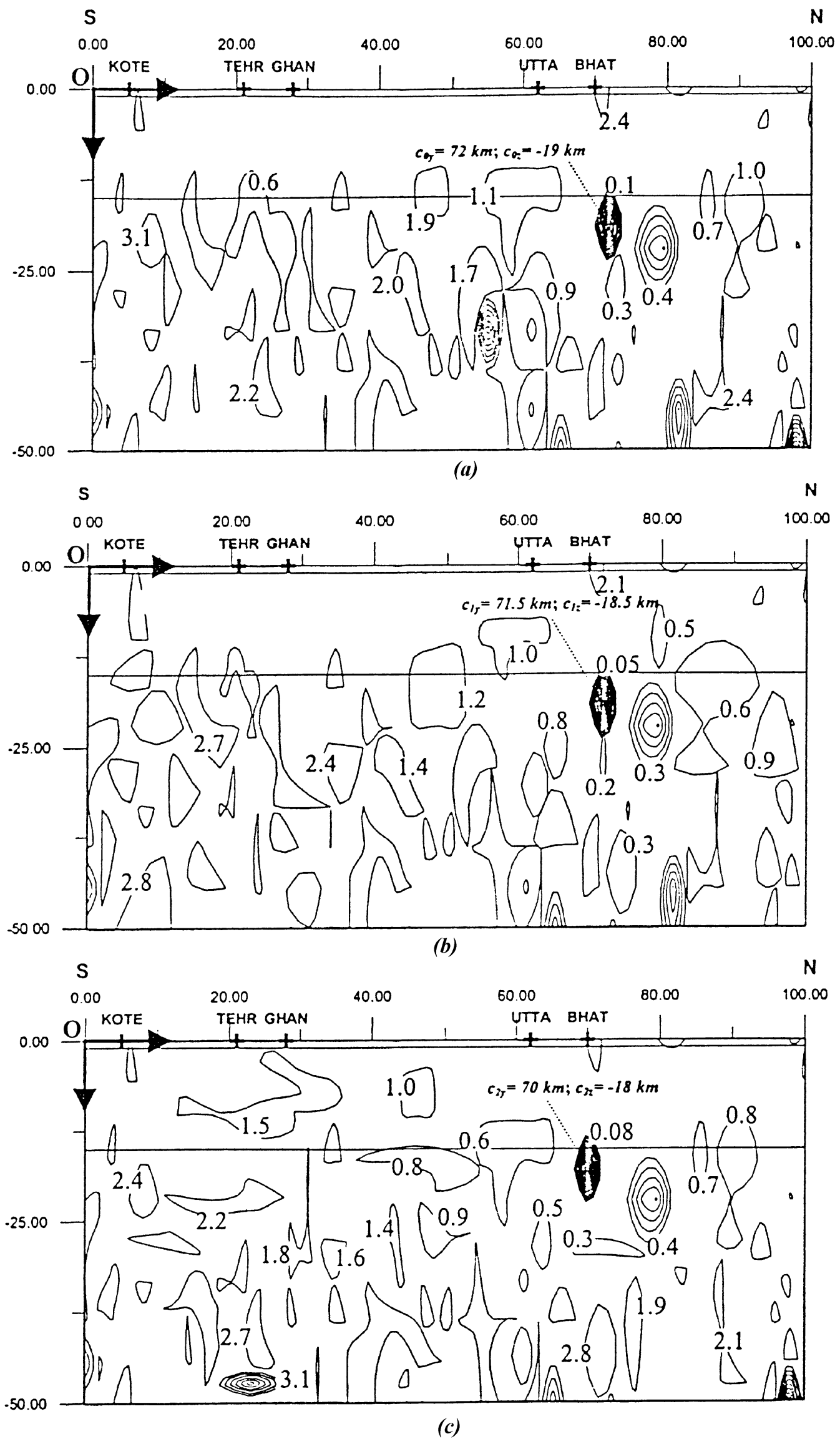

Figure 5. Contours of mean square error (a) 0-0.5 sec (b) 0.5-1 sec (c) 1-1.5 sec. 
mean square error in the equation. First, the approach will be indicated for the case of a single impulse applied at $t=s=0$. The surface response for this case, valid for all $t$ is

$$
\begin{aligned}
u_{i}(t) & =I_{0 x} P_{u, i 0}(t)+I_{0 z} Q_{u, i 0}(t), \\
w_{i}(t) & =I_{0 x} P_{w, i 0}(t)+I_{0 z} Q_{w, i 0}(t) .
\end{aligned}
$$
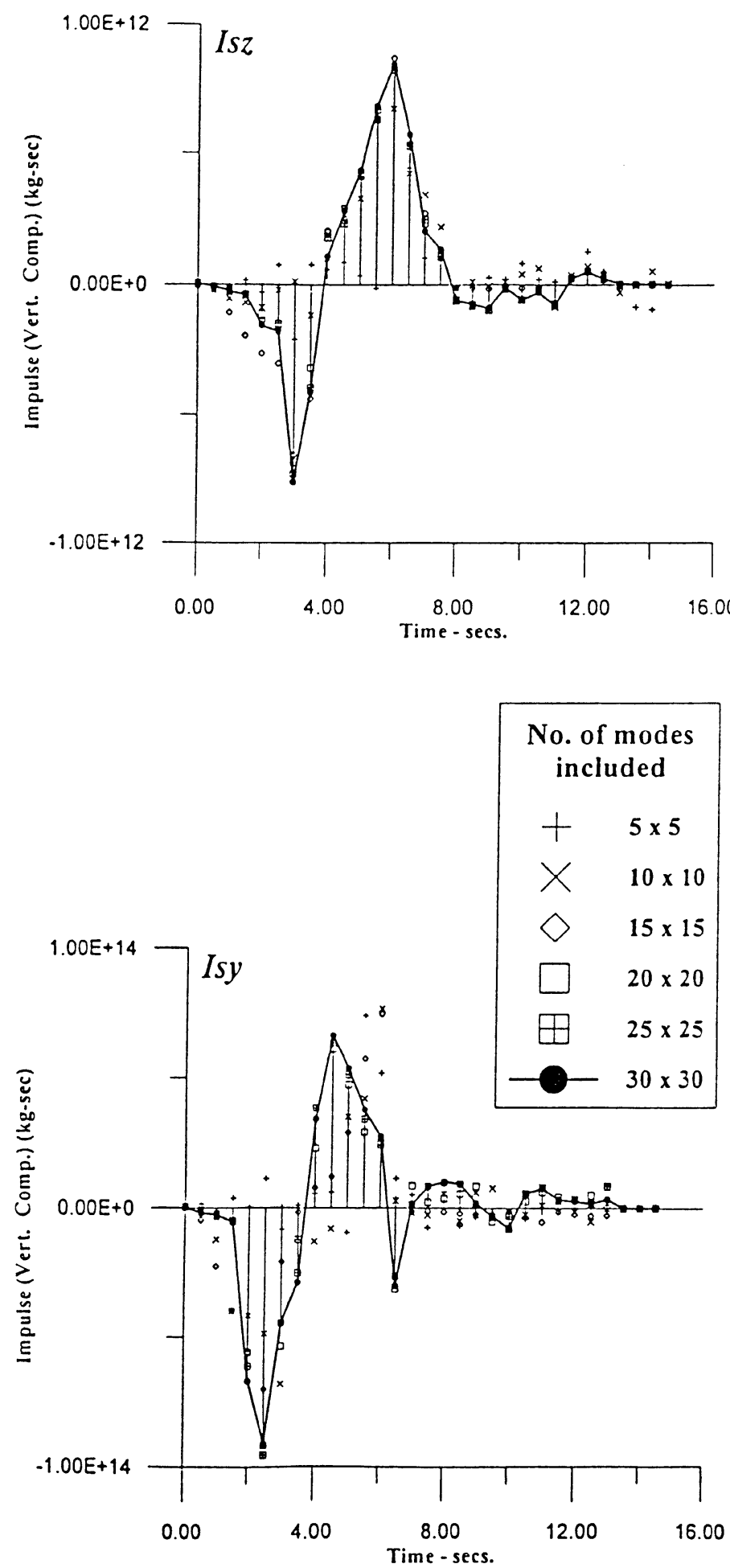

Figure 6. Convergence of source impulse and displacements.

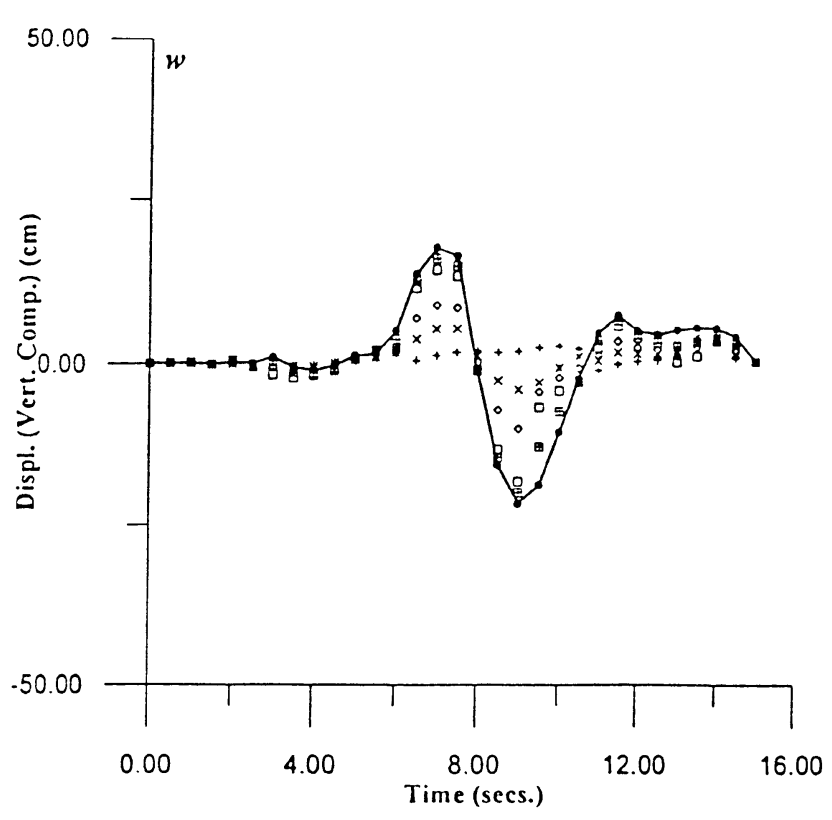

The total mean square error over the interval of measurement $(0, T)$ is

$$
\begin{aligned}
E^{2}=\sum_{i=1}^{p} E_{i}^{2}= & \sum_{i=1}^{p} \int_{0}^{T}\left[u_{i}-I_{0 x} P_{u, i 0}-I_{0 z} Q_{u, i 0}\right]^{2} \\
& +\left[w_{i}-I_{0 x} P_{w, i 0}-I_{0 z} Q_{w, i 0}\right]^{2}
\end{aligned}
$$

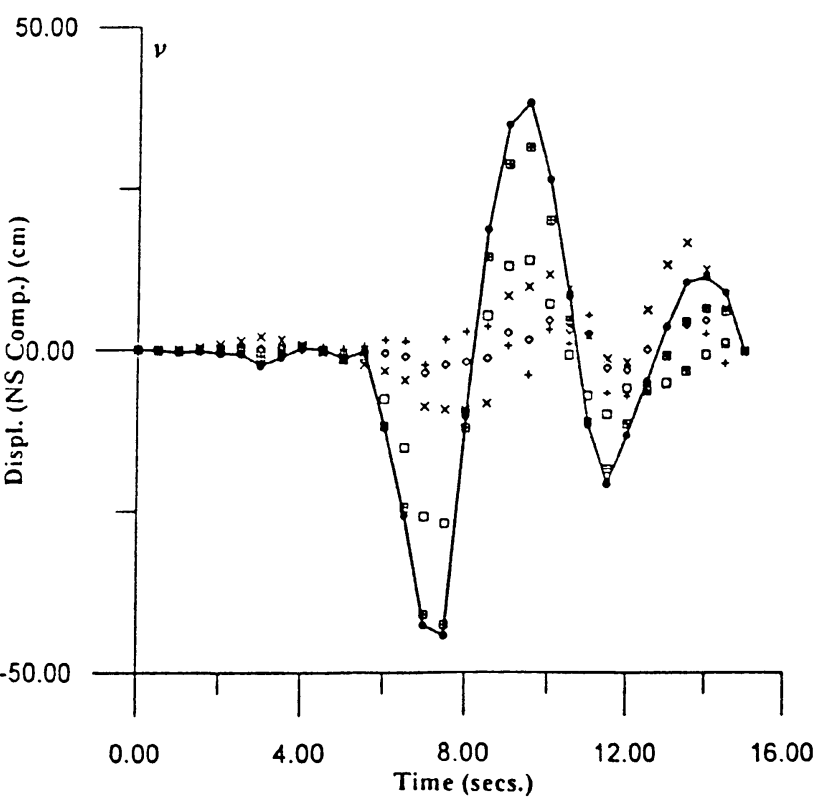


From the minimizing conditions,

$$
\frac{\partial E^{2}}{\partial I_{0 x}}=\frac{\partial E^{2}}{\partial I_{0 z}}=0
$$

One easily gets

$$
\begin{aligned}
& I_{0 x} g_{11}+I_{0 z} g_{12}=S_{1}, \\
& I_{0 x} g_{21}+I_{0 z} g_{22}=S_{2} .
\end{aligned}
$$

Where, the coefficients $g_{11}, g_{12}, g_{21}, g_{22}, S_{1}, S_{2}$ still contain the unknowns $\left(c_{0 x}, c_{0 z}\right)$. Error minimization w.r.t. $c_{0 x}, c_{0 z}$ is possible; however simultaneous solution of all the equations is numerically cumbersome. An alternate error criterion to find $\left(c_{0 x}, c_{0 z}\right)$ is as follows.

From equations (18) and (19), one can write

$$
\begin{gathered}
I_{0 x}=\left(u_{i} Q_{w, i 0}-w_{i} Q_{u, i 0}\right) / \Delta_{i}=R_{i x} / \Delta_{i}, \\
I_{0 z}=\left(-u_{i} P_{w, i 0}+w_{i} P_{u, i 0}\right) / \Delta_{i}=R_{i z} / \Delta_{i}, \\
\Delta_{i}=\left(P_{u, i 0} Q_{w, i 0}-P_{w, i 0} Q_{u, i 0}\right) .
\end{gathered}
$$

Since, the above is to be valid for every $i$, it follows

$$
\begin{aligned}
R_{1 x} \Delta_{2} & =R_{2 x} \Delta_{1} ; R_{2 x} \Delta_{3} \\
& =R_{3 x} \Delta_{2} ; \ldots R_{p-1 x} \Delta_{p}=R_{p x} \Delta_{p-1},
\end{aligned}
$$

$$
\begin{aligned}
R_{1 z} \Delta_{2} & =R_{2 z} \Delta_{1} ; R_{2 z} \Delta_{3} \\
& =R_{3 z} \Delta_{2} ; \ldots R_{p-1 z} \Delta_{p}=R_{p z} \Delta_{p-1} .
\end{aligned}
$$

The total mean square error will be

$$
\begin{aligned}
\varepsilon^{2}= & \sum_{i=1}^{p-1} \int_{0}^{T}\left[\left(R_{i x} \Delta_{i+1}-R_{i+1 x} \Delta_{i}\right)^{2}\right. \\
& \left.+\left(R_{i z} \Delta_{i+1}-R_{i+1 z} \Delta_{i}\right)^{2}\right] \mathrm{d} t .
\end{aligned}
$$

One can easily find the value of $\left(c_{0 x}, c_{0 z}\right)$ for which $\varepsilon^{2}$ will be the absolute minimum in the domain of the

\begin{tabular}{|c|c|c|c|c|c|c|c|}
\hline \multirow[t]{2}{*}{ Interval $S$} & \multirow[t]{2}{*}{ Time, sec. } & \multicolumn{2}{|c|}{ Location, km } & \multicolumn{2}{|c|}{ Impulse, kg-sec } & \multicolumn{2}{|c|}{ Displacement, cm } \\
\hline & & $C_{s y}$ & $C_{s z}$ & $I_{s y}$ & $I_{s z}$ & $v$ & $w$ \\
\hline 0 & $0-0.5$ & 72 & -19 & $1.61 \mathrm{E}+11$ & $3.06 \mathrm{E}+09$ & 0.000 & 0.000 \\
\hline 1 & $0.5-1.0$ & 71.5 & -18.5 & $-1.89 \mathrm{E}+12$ & $-7.00 \mathrm{E}+09$ & -0.099 & 0.050 \\
\hline 2 & $1.0-1.5$ & 70 & -18 & $-2.59 \mathrm{E}+12$ & $-2.60 \mathrm{E}+10$ & -0.358 & 0.034 \\
\hline 3 & $1.5-2.0$ & 69.5 & -17.5 & $-5.38 \mathrm{E}+12$ & $-3.90 \mathrm{E}+10$ & -0.098 & -0.139 \\
\hline 4 & $2.0-2.5$ & 68 & -17 & $-6.71 \mathrm{E}+13$ & $-1.56 \mathrm{E}+11$ & -0.476 & 0.059 \\
\hline 5 & $2.5-3.0$ & 67 & -16.5 & $-9.14 \mathrm{E}+13$ & $-1.78 \mathrm{E}+11$ & -0.557 & -0.027 \\
\hline 6 & $3.0-3.5$ & 66 & -16 & $-4.47 \mathrm{E}+13$ & $-7.66 \mathrm{E}+11$ & -2.345 & 0.927 \\
\hline 7 & $3.5-4.0$ & 64 & -16 & $-2.87 \mathrm{E}+13$ & $-4.17 \mathrm{E}+11$ & -0.955 & -0.590 \\
\hline 8 & $4.0-4.5$ & 63 & -16 & $3.42 \mathrm{E}+13$ & $1.08 \mathrm{E}+11$ & 0.355 & -1.075 \\
\hline 9 & $4.5-5.0$ & 62.5 & -15.5 & $6.64 \mathrm{E}+13$ & $2.80 \mathrm{E}+11$ & 0.198 & -0.390 \\
\hline 10 & $5.0-5.5$ & 61 & -14.5 & $5.37 \mathrm{E}+13$ & $4.33 \mathrm{E}+11$ & -1.112 & 1.036 \\
\hline 11 & $5.5-6.0$ & 59 & -14.5 & $3.77 \mathrm{E}+13$ & $6.82 \mathrm{E}+11$ & 0.021 & 1.328 \\
\hline 12 & $6.0-6.5$ & 57 & -14 & $2.74 \mathrm{E}+13$ & $8.41 \mathrm{E}+11$ & -11.755 & 4.807 \\
\hline 13 & $6.5-7.0$ & 54 & -13.5 & $-3.00 \mathrm{E}+13$ & $5.71 \mathrm{E}+11$ & -25.467 & 13.567 \\
\hline 14 & $7.0-7.5$ & 53 & -13 & $1.11 \mathrm{E}+12$ & $2.07 \mathrm{E}+11$ & -42.703 & 17.573 \\
\hline 15 & $7.5-8.0$ & 60 & -16 & $8.11 \mathrm{E}+12$ & $1.36 \mathrm{E}+11$ & -44.321 & 16.319 \\
\hline 16 & $8.0-8.5$ & 57.5 & -14.5 & $9.97 \mathrm{E}+12$ & $-6.14 \mathrm{E}+10$ & -10.004 & -1.299 \\
\hline 17 & $8.5-9.0$ & 56 & -13.5 & $9.16 \mathrm{E}+12$ & $-7.52 \mathrm{E}+10$ & 18.902 & -15.802 \\
\hline 18 & $9.0-9.5$ & 46 & -12.5 & $1.87 \mathrm{E}+12$ & $-9.08 \mathrm{E}+10$ & 35.043 & -21.819 \\
\hline 19 & $9.5-10.0$ & 44 & -12.5 & $-3.11 \mathrm{E}+12$ & $-1.21 \mathrm{E}+10$ & 38.374 & -18.989 \\
\hline 20 & $10.0-10.5$ & 47 & -12.5 & $-7.54 \mathrm{E}+12$ & $-5.82 \mathrm{E}+10$ & 26.547 & -10.651 \\
\hline 21 & $10.5-11.0$ & 43.5 & -12 & $5.40 \mathrm{E}+12$ & $-2.90 \mathrm{E}+10$ & 8.528 & -2.530 \\
\hline 22 & $11.0-11.5$ & 42 & -12 & $7.52 \mathrm{E}+12$ & $-7.99 \mathrm{E}+10$ & -11.364 & 4.588 \\
\hline 23 & $11.5-12.0$ & 41.5 & -11.5 & $3.09 \mathrm{E}+12$ & $2.44 \mathrm{E}+10$ & -20.696 & 7.367 \\
\hline 24 & $12.0-12.5$ & 41 & -11.5 & $2.64 \mathrm{E}+12$ & $4.94 \mathrm{E}+10$ & -13.039 & 4.930 \\
\hline 25 & $12.5-13.0$ & 40.5 & -12 & $1.69 \mathrm{E}+12$ & $2.21 \mathrm{E}+10$ & -4.636 & 4.379 \\
\hline 26 & $13.0-13.5$ & 41 & -11 & $3.33 \mathrm{E}+12$ & $3.94 \mathrm{E}+09$ & 3.659 & 5.034 \\
\hline 27 & $13.5-14.0$ & 42.5 & -11 & $3.30 \mathrm{E}+10$ & $3.25 \mathrm{E}+09$ & 10.679 & 5.367 \\
\hline 28 & $14.0-14.5$ & 44.5 & -11 & $-1.44 \mathrm{E}+10$ & $3.47 \mathrm{E}+09$ & 11.580 & 5.287 \\
\hline 29 & $14.5-15.0$ & 40.5 & -12 & $-1.20 \mathrm{E}+10$ & $1.42 \mathrm{E}+09$ & 0.000 & 0.000 \\
\hline
\end{tabular}
medium. With this value of $\left(c_{0 x}, c_{0 z}\right)$, one can solve equation (22) to find the values of $\left(I_{0 x}, I_{0 z}\right)$ as per the present approach.

\section{Recursive estimation of $I_{s}$ and $c_{s}$}

Let the source impulse at $t=0$ be known by the above method considering a small interval $\left(0, t_{1}\right)$. With the help of these estimated values, the response for the next time interval can be written as superposition of a known response $\left(\hat{u}_{i 0}, \hat{w}_{i 0}\right)$ and a new component due to the impulse at $t=t_{1}$. Thus, for every station $i$, in

Table 1. Source location, impulse magnitude and displacements ( $N-S$ section, Uttarkashi earthquake). 
$\left(t_{1} \leq t<t_{2}\right)$

$$
\begin{aligned}
{\left[u_{i}(t)-\hat{u}_{i 0}(t)\right] } & =\left(I_{1 x} P_{u, i 1}+I_{1 z} Q_{u, i 1}\right), \\
{\left[w_{i}(t)-\hat{w}_{i 0}(t)\right] } & =\left(I_{1 x} P_{w, i 1}+I_{1 z} Q_{w, i 1}\right) .
\end{aligned}
$$

Following the same procedure as previously explained, the unknowns $\left(I_{1 x}, I_{1 z}\right)$ can be found by minimizing the mean square error between the LHS and RHS of the above equations. The location of new impulse is estimated using an error criterion similar to equation (28), which does not contain $\left(I_{1 x}, I_{1 z}\right)$. It is to be noted here that responses $\left(u_{i}, w_{i}\right)$ are measured or recorded values where as $\left(\hat{u}_{i 0}, \hat{w}_{i 0}\right)$ are found for the interval under consideration from the estimated

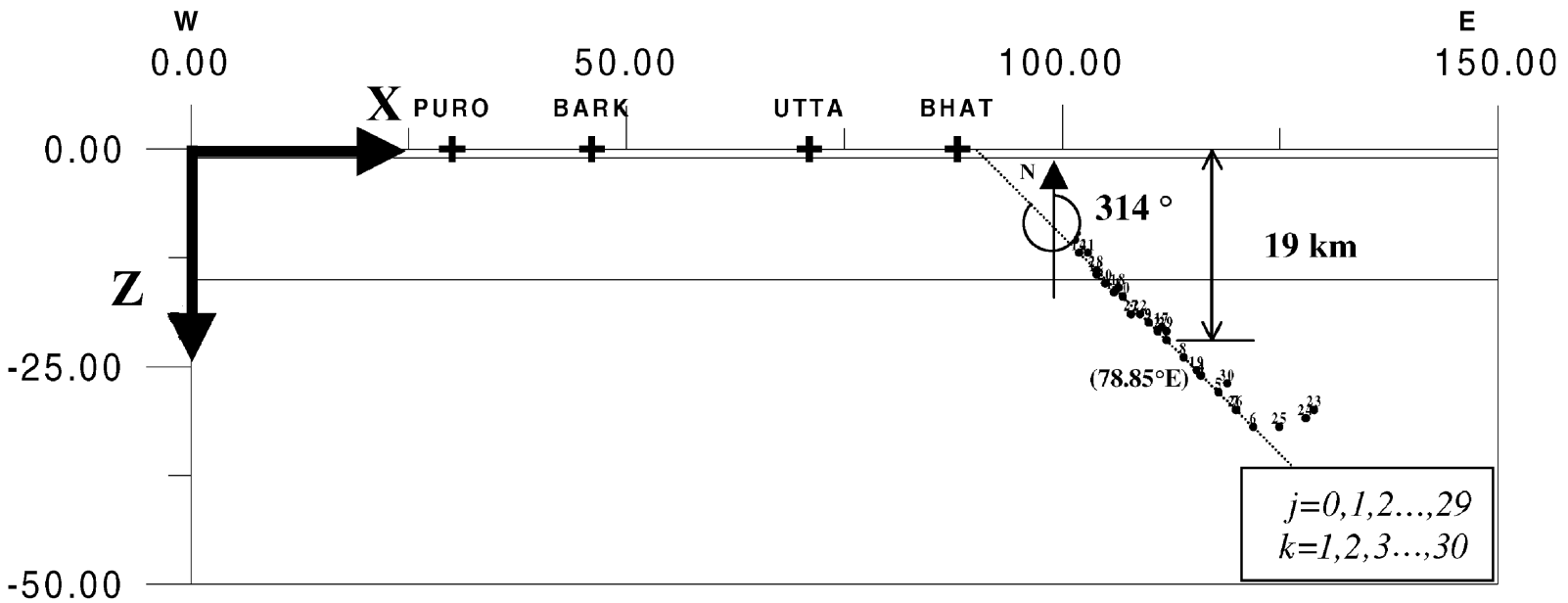

(a)

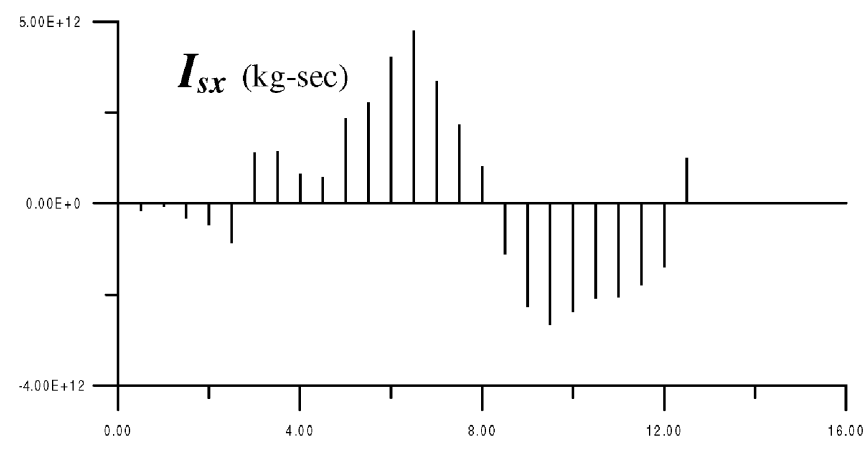

Time (sec)

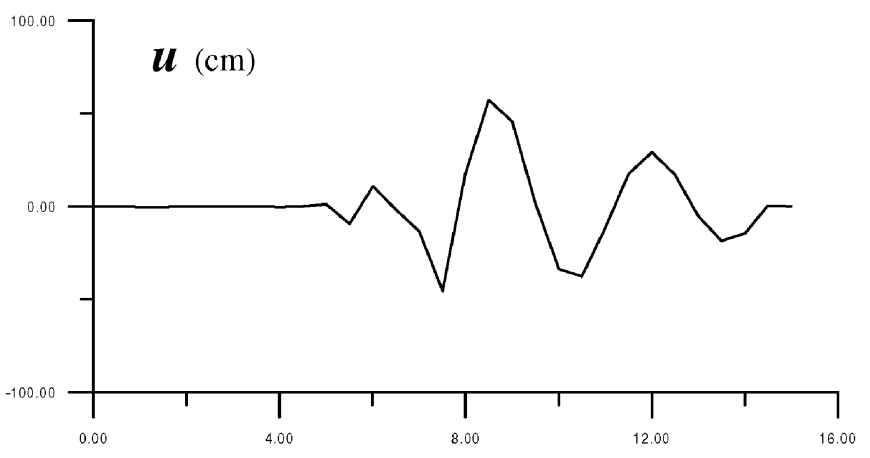

Time (sec)

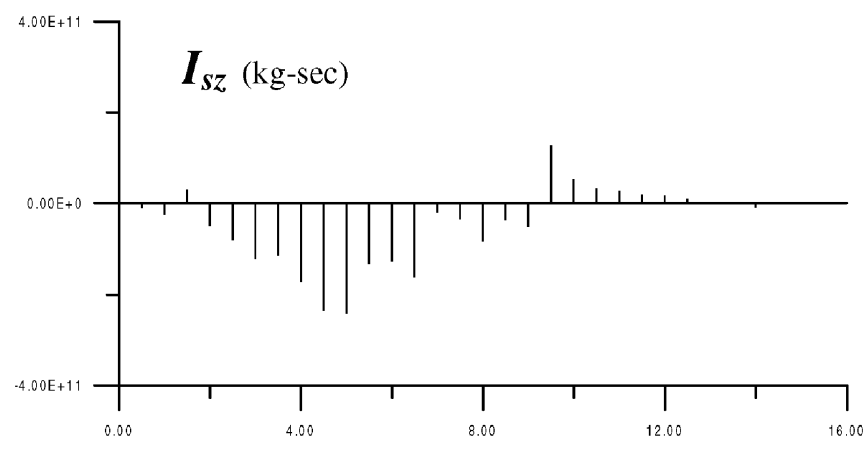

(b)

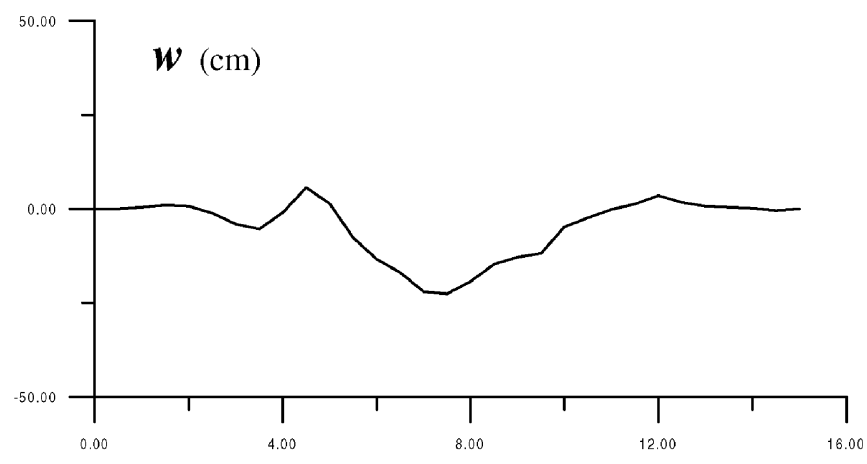

Time (sec)

(c)

Figure 7. Source location, magnitude of equivalent impulses and displacement at the souce (EW section, Uttarkashi earthquake). 
source for the previous interval $\left(0, t_{1}\right)$. Thus, we have a time marching method to find $\left(I_{s x}, I_{s z}\right)$ and $\left(c_{s x}, c_{s z}\right)$ in the time interval $\left(t_{s} \leq t<t_{s+1}\right)$ in terms of already estimated values of $\left(I_{0 x}, I_{0 z}\right) ;\left(I_{1 x}, I_{1 z}\right) ; \ldots\left(I_{s-1 x}, I_{s-1 z}\right)$ and $\left(c_{0 x}, c_{0 z}\right) ;\left(c_{1 x}, c_{1 z}\right) ; \ldots\left(c_{s-1 x}, c_{s-1 z}\right)$.

\section{Numerical results}

The above method has been applied to the Uttarkashi event, using the ground displacement records of a few stations. With reference to figure 3 , a section is taken in the N-S direction such that this passes through Bhatwari, which recorded the largest displacements during the 1991 earthquake. Four other stations which are very close to this section are presumed to be on the surface as shown in figure 4 . This is a limitation of the two-dimensional model used here, which can be overcome only by analyzing a 3 -D elastic medium. The natural frequencies and mode shapes of the threelayered regional model have been found. The N-S and vertical displacement components of the five stations indicated above have been inverted using the method described previously. The total length of the record is 15 sec. The time step for impulse and source determination has been chosen as 0.5 sec uniformly. Since, the response is expressed as a series (equations 4,5 ), it is necessary to include all terms which contribute in the frequency range of interest namely 0-0.5 Hz. In the present problem, it is found that this includes all modes with $(j=0,1,2, \ldots 29)$ and $(k=1,2, \ldots 30)$. To see how the number of included modes influences the source picture, the convergence of $\left(c_{s x}, c_{s z}\right)$ is shown in figure 5 . In all the cases, the contours of $\left(\varepsilon^{2}\right)$ are plotted on a grid of $0.5 \times 0.5 \mathrm{~km}$ to find the error minima. A typical picture of the error contour is shown in figure 5, for arriving at the final results of figure 4(f). Along with the source location in time, the corresponding impulse values and displacements are also obtained. These results are shown in figure 6 for both the N-S and vertical directions.

For clarity, the final numerical results obtained with $(30 \times 30)$ terms are presented in table 1 . It is observed that the first impulse is located at a depth of $19 \mathrm{~km}$, which will be the estimated focal depth also. Since, the present model uses a 2-D section, for the Himalayan region $\mathrm{N}-\mathrm{S}$ and $\mathrm{E}-\mathrm{W}$ direction would be the natural choice. The final results obtained for the E-W section passing through Bhatwari are shown in figure 7 .

\section{Simulation}

In many engineering problems, simulation of ground acceleration time history is required. Accelerograms contain high frequencies up to the order of $10 \mathrm{~Hz}$ and more. How good is the present source model in simulating the recorded accelerations at the stations? To answer this question, the impulse source model

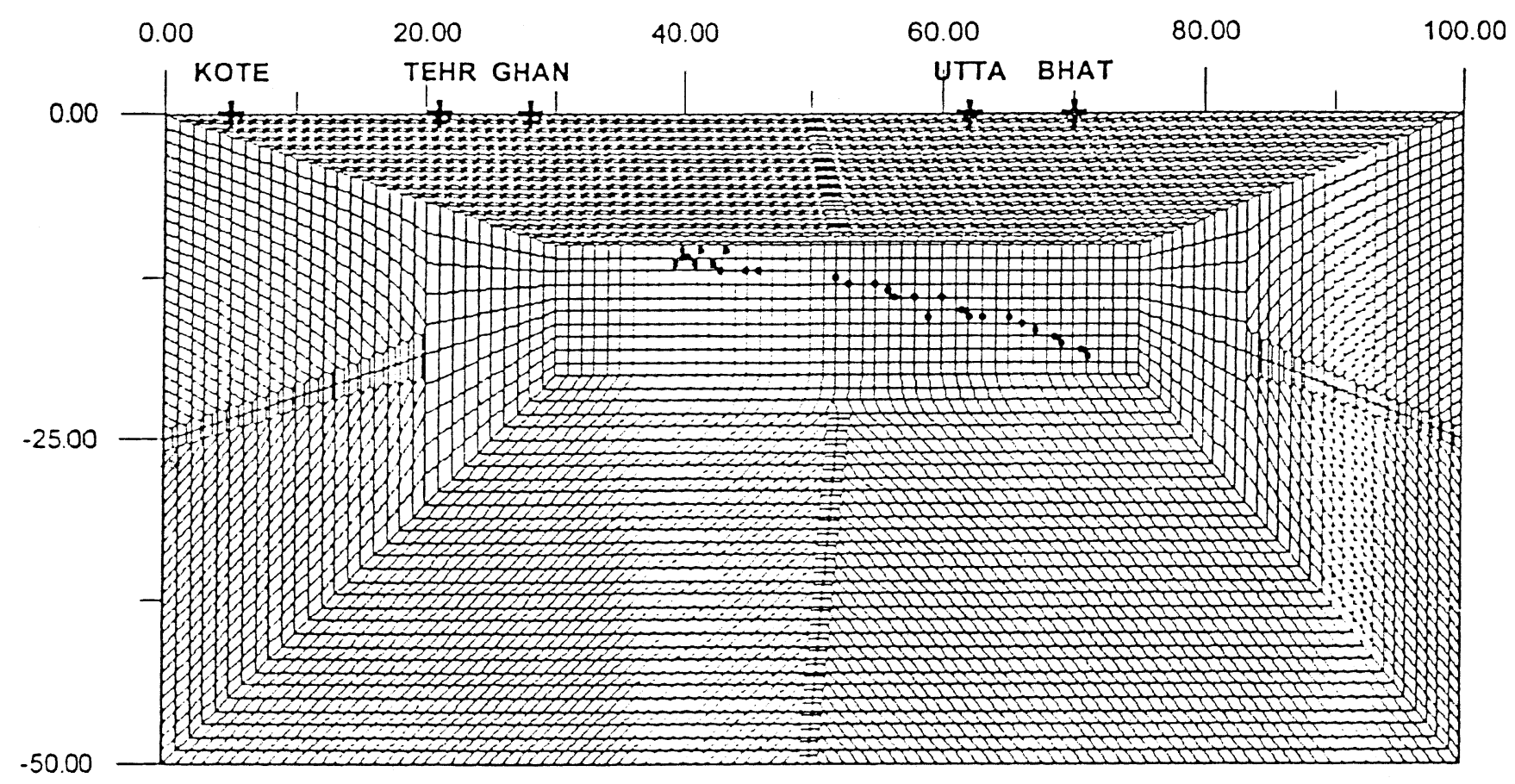

largest element : $1 \mathrm{kmx} 1 \mathrm{~km}$ smallest element : $100 \mathrm{~m} \times 100 \mathrm{~m}$

Figure 8. Finite element discretisation of three layered model (NS section). 


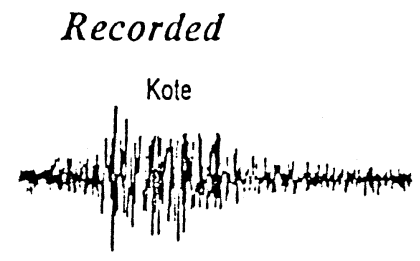

Simulated

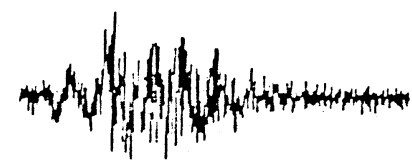

mand
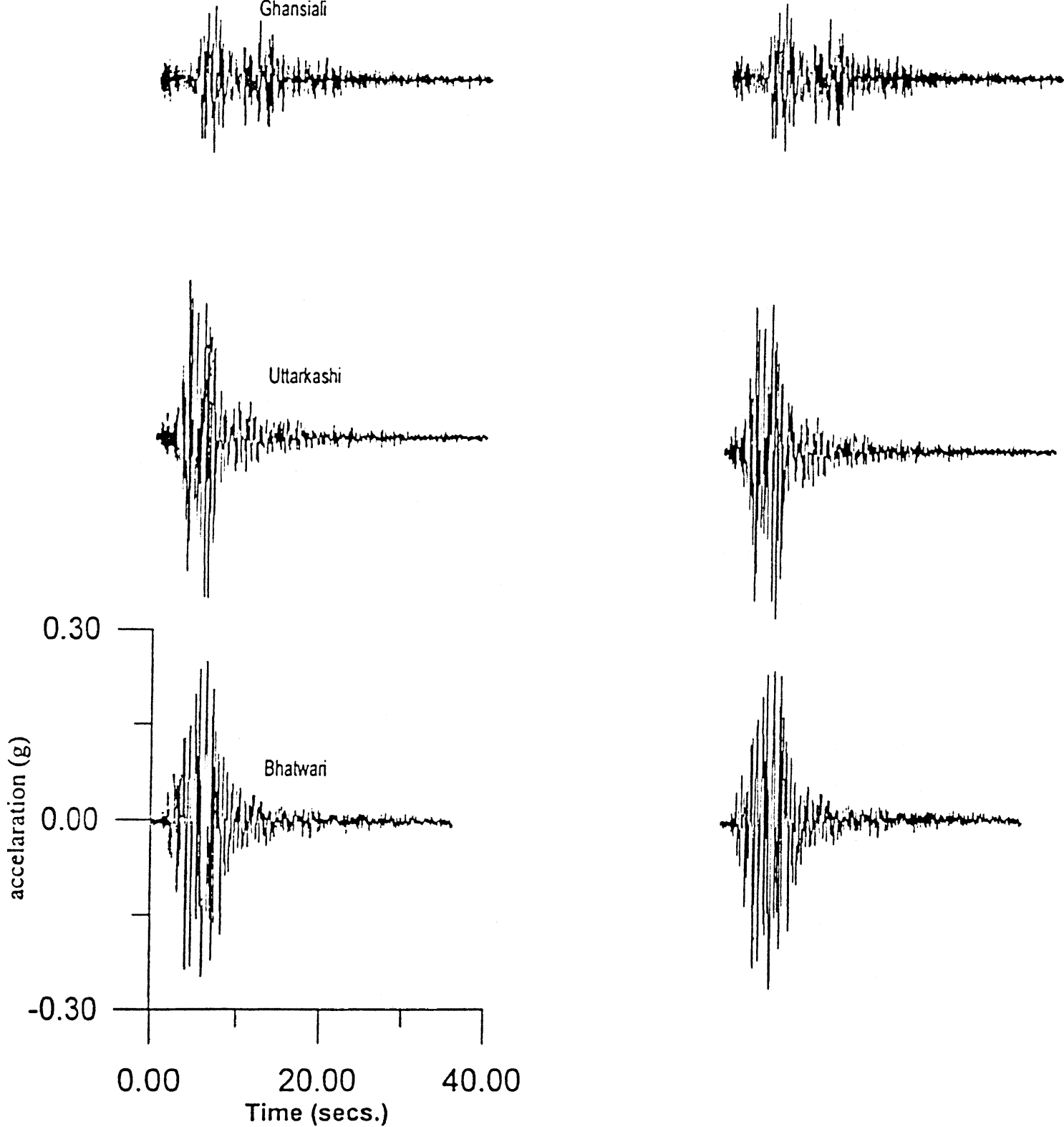

Figure 9. Comparison of acceleration time histories for Uttarkashi event (NS section). 
of table 1 is used to find the forced response of the 3-layered regional medium by finite element methods. In figure 8 , the FEM discretization of the medium using a graded mesh is shown for use with standard commercial software NISA II (1994). The estimated impulses are applied in a time sequence at the
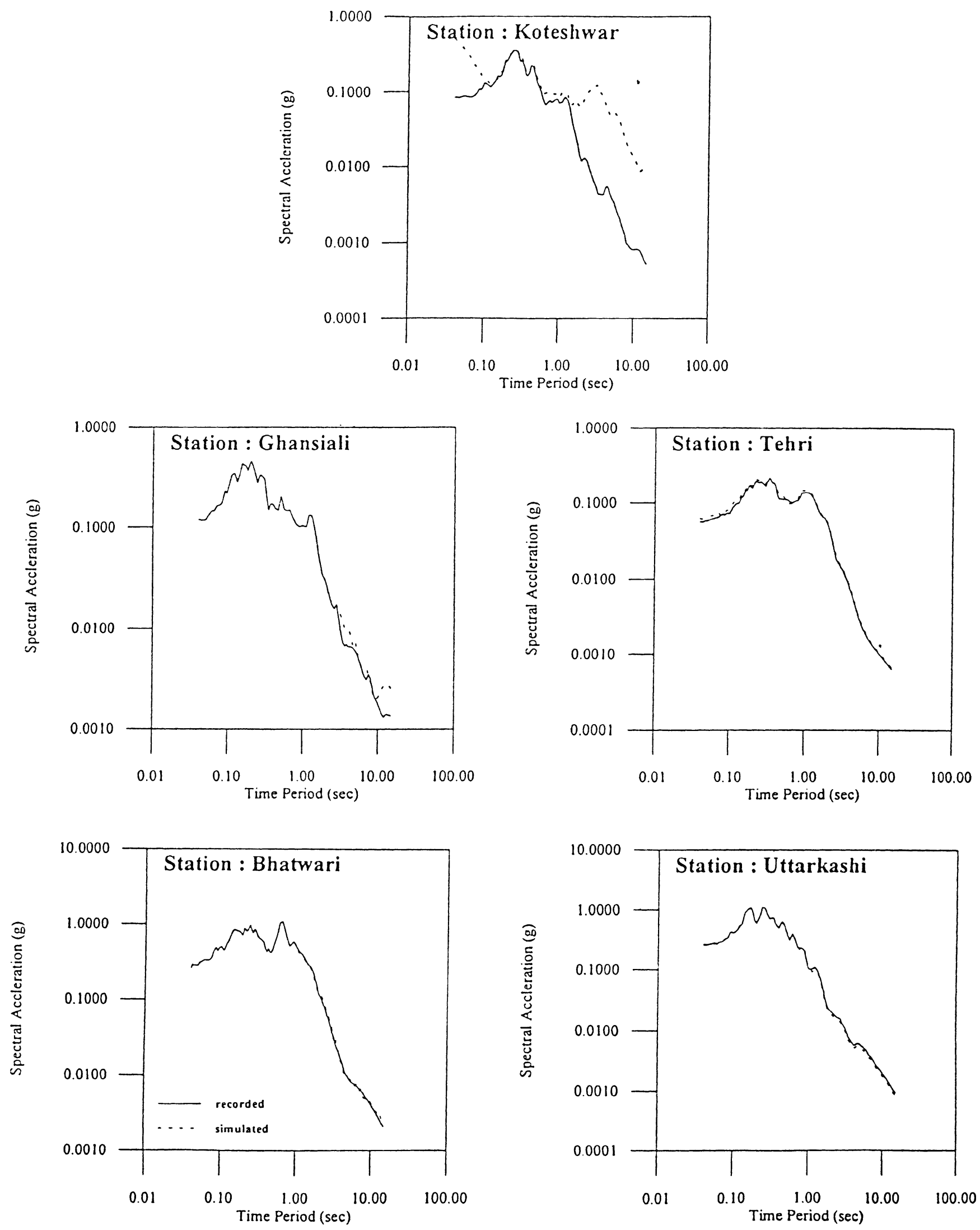

Figure 10. Response spectra at 5\% damping (NS Comp.). 
indicated locations. The first five hundred modes reaching up to $10 \mathrm{~Hz}$ are used in the forced response analysis. The surface accelerations as obtained by NISA II at the five stations are compared with the actually recorded accelerograms in figure 9 . The corresponding response spectra, which are more popular with engineers, are presented in figure 10.

\section{Discussion and conclusions}

The results obtained above can be interpreted as a simulated engineering model for the earthquake source. The consistency and contiguous nature of the impulse locations obtained indicate that these points should be on the fault sections. The linear structure of the source points further support this observation. From each of the two sections, two different values of the depth are obtained. However, these values are nearly the same in the initial period which again is an indication of the robustness of the proposed approach. For the data considered here, the depth of the first impulse is obtained as $19 \mathrm{~km}$ from both the N-S and E-W regional models. The $\left(c_{0 x}, c_{0 y}\right)$ values obtained from two sections can be interpreted as the location of the epicentre. For the present case, this turns out to be $30.74^{\circ} \mathrm{N}$ and $78.85^{\circ} \mathrm{E}$. This point is almost the same as the epicentre reported by GSI (1992). The focal depth obtained here compares closely with the value reported by USGS. The dip angle of the source line in the N-S section in figure $4(\mathrm{f})$ is $14^{\circ}$. Similarly for the E-W section, the strike is estimated to be $314^{\circ}$ (figure 7 ). These compare well with the previous values reported in the literature $(\mathrm{Yu}$ et al 1995). The impulses $I_{s}$ found at the source can be interpreted as equivalent forces $\left(I_{s} / \Delta t\right)$, where $\Delta t$ is the time step. In the present analysis, $\Delta t=0.5 \mathrm{sec}$. The total source energy can be easily estimated as the sum of the work done by the forces at all the locations. Hence, for the E-W direction, $W_{E W}=\sum_{s} I_{s x} u_{s} / \Delta t$. For the Uttarkashi event, the present approach gives $W_{E W}=1.12 \times 10^{21}$ ergs. Similarly in the N-S direction, one gets $W_{N S}=3.12 \times 10^{21}$ ergs. For the vertical direction, two values $W_{V(E W)}=1.94 \times 10^{21}$ ergs; $W_{V(N S)}=2.01 \times 10^{21} \mathrm{ergs}$, which are almost same, are obtained. The total work done as per the present engineering model comes out to be $4.35 \times 10^{21} \mathrm{ergs}$, whereas the value obtained by geophysical methods is $4.36 \times 10^{21} \mathrm{ergs}$ (GSI 1992). The simulated accelerograms and response spectra of figures 10 and 11 compare well with the actually recorded values at the interior stations away from the boundaries. The poor comparison at station Koteshwar is clearly attributable to its nearness to the artificial boundary used in the present regional model. The other limitation of the model is the representation of the fault rupture as a sequence of equivalent single forces, instead of the more appropriate double couples. However, these limitations can be overcome within the frame work of the limited area regional model at greater computational effort. Improvements of the proposed method to include three dimensional effects and double couples as source functions will be reported in a future publication.

\section{References}

Chandrasekaran A R and Das J D 1995 Strong motion records from Uttarkashi earthquake, Mem. Geol. Soc. India, 30 pp. 133-147

Ewing W M, Jardetzky W S and Press F 1957 Elastic waves in Layered Media, (McGraw-Hill Book Company Inc.,)

Geological Survey of India 1992 Uttarkashi Earthquake October, 20, 1991 (1992), Special Publication No. 30.

Iyengar R N and Iyengar K T S 1969 'A non-stationary random process model for earthquake accelerograms,' Bulletin of the Seismological Society of America, 59 pp. 1163-1188

Iyengar R N and Rao P N 1975 Free vibration of an elastic medium; Bulletin of the Indian Society of Earthquake Technology, 12(4) December 1975, pp. 147-154

Khattri K N 1993 An overview of seismicity of Himalaya, In Earthquake Hazard and Large Dams in the Himalaya, (ed) V K Gaur, New Delhi: INTACH

Khattri K N, Yu G, Anderson J G, Brune J N and Zeng Y 1994 Seismic hazard estimation using modelling of earthquake strong ground motions: a brief analysis of 1991 Uttarkashi earthquake, Himalaya and prognostication for a great earthquake in the region, Curr. Sci. 67(5), September, pp. 343-353

Kumar D Khattri K N Teotia S S and Rai S S 1999 Modelling of accelerograms of two Himalayan earthquakes using a novel semi-empirical method and estimation of accelerogram for a hypothetical great earthquake in the Himalaya; Curr. Sci. 76(6) 25 March 1999, pp. 819-830

NISA II 1994 User's Manual, Engineering Mechanics Research Corporation, Troy, Michigan, USA, December 1994.

Shinozuka H and Sato Y 1967 Simulation of non-stationary random process; Journal of Engineering Mechanics Division ASCE, 93(1), pp. 11-40

Yu G, Khattri K N, Anderson J G, Brune J N and Zeng Y 1995 Strong ground motion from the Uttarkashi, Himalaya, India, earthquake: Comparison of observations with synthetics using the composite source model; Bulletin of the Seismological Society of America, 85(1), February, pp. $31-50$ 